\title{
Leishmaniose tegumentar americana no Distrito Federal
}

\author{
Mucocutaneous leishmaniasis in the Federal District
}

Raimunda Nonata Ribeiro Sampaio e Carmen Déa Ribeiro de Paula

\begin{abstract}
Resumo Relatamos aqui 11 casos de leishmaniose tegumentar americana (LTA) em pacientes que residem no DF e que não saíram da sua área durante um tempo que variou de seis meses a dois anos antes do início da doença. Seis dos 11 pacientes, residem na cidade satélite de Planaltina. Todos têm a intradermorreação de Montenegro positiva. Dez deles têm presença de leishmânia nas lesões. Nas lesões de dois pacientes foram identificadas, pelo método de anticorpos monoclonais, Leishmania (V) braziliensis. Nove deles, foram tratados com antimonial pentavalente e dois com pentamidina. Houve duas ocorrências de recidiva, ambas, após o uso do antimonial. Constatada a presença de vetores e de pacientes infectados no Distrito Federal, acredita-se que possa estar ocorrendo infecção por leishmânia em Brasília e em suas áreas periurbanas.
\end{abstract}

Palavras-chaves: Leishmaniose tegumentar american. Leishmaniose cutâneo-mucosa. Epidemiologia. Brasília. Distrito Federal.

Abstract In the present study, we report 11 cases of mucocutaneous leishmaniasis (MCL) in patients living in the Federal District who had not left their area of residence for a period ranging from six months to two years before the onset of the disease. Six of the eleven patients live in the suburban town of Planaltina. All showed a positive Leishmanin intradermal reaction. Ten of them presentedt the parasite in their lesions. Leishmania (V) braziliensi was identified in the lesions of two patients by the monoclonal antibody method. Nine patients were treated with pentavalent antimoy and two with pentamidine. Relapse occurred in two cases after treatment with antimony. In view of the detection of vectors and infected patients in the Federal District, it is probable that Leishmania infection is occurring in Brasilia and its suburban areas.

Key-words: Mucocutaneous leishmaniasis. Epidemiology. Brasília. Distrito Federal.

A leishmaniose tegumentar americana (LTA) consiste de zoonose endêmica, de difícil controle, cujas estatísticas oficiais mostram no Brasil, nas últimas décadas, crescimento progressivo e registros ocasionais de surtos epidêmicos ${ }^{815}$. Dentre as cinco regiões brasileiras, a Centro-Oeste figura como a terceira em incidência e a primeira em crescimento da doença, aumentando de 130 casos em 1980 para 4.658 em 1996, segundo dados do Ministério da Saúde (MS) ${ }^{8}$.

A doença apresenta uma grande variedade de manifestações clínicas. A forma mais comum, é representada por uma úlcera única que pode cicatrizar espontaneamente, entretanto, algumas vezes, pode causar lesões de mucosas nasal, oral, faringe e laringe podendo $5 \%$ destes pacientes evoluir para o óbito por complicações, como infecção ${ }^{13}$.

Grandes nomes da Medicina Brasileira contribuíram para o estudo da leishmaniose e, desde então, muitos estudos têm sido realizados no campo microbiológico e terapêutico, como também muitos estudos epidemiológicos foram realizados nas regiões de povoamento mais

Departamento de Clínica Médica, Faculdade de Ciências da Saúde da Universidade de Brasília, Brasília, DF.

Endereço para correspondência: Prof ${ }^{\underline{a}}$ Raimunda Nonata Ribeiro Sampaio. Laboratório de Micologia/Dept ${ }^{\circ}$ de Clínica Médica/FCS/ UnB, Campus Universitário, 70919-900 Brasília, DF. Fax: 55 61 307-2502.

Recebido para publicação em 18/9/99. 
antigo como São Paulo, Rio de Janeiro, Minas Gerais, bem como a Região Amazônica e o interior da Bahia4911 12141524 .

\section{MATERIAL E MÉTODOS}

Local de estudo: O Distrito Federal (DF) ocupa uma área de $58.114 / \mathrm{km}^{2}$, localizado na

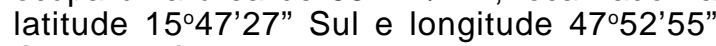
Oeste. Está totalmente contido dentro do estado de Goiás, exceto por estreita faixa no Sudeste onde faz fronteira com o estado de Minas Gerais. Consiste em uma área de povoamento recente, com 37 anos de existência e crescimento populacional contínuo. Tem altitude de $1.100 \mathrm{~m}$, com ponto culminante a $1.349 \mathrm{~m}$. Possui um clima quente e seco com temperatura variando de 15$25,8^{\circ} \mathrm{C}$, e índice pluviométrico de $1.675 \mathrm{~mm}$ anuais, estando $80 \%$ das chuvas concentradas de novembro a abril. Sua vegetação predominante é o cerrado entrecortado por matas ciliares.

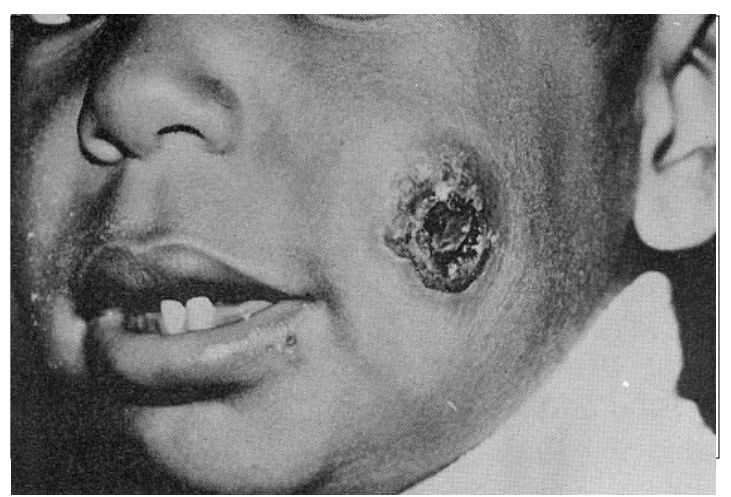

Figura 1 - Lesão ulcerada crostosa, com presença de amastigotas pelo esfregacco, em face de criança de um ano satélite de Planaltina).
Pretendemos apresentar neste trabalho, 11 casos de LTA que nos parecem autóctones do Distrito Federal.

Pacientes. Selecionamos 11 pacientes, todos residentes no DF e que não saíram da sua área durante um tempo que variou de seis meses a dois anos. Uma criança de um ano e três meses nasceu aqui, e nunca havia saído do DF (Figuras 1, 2 e 3). Todos apresentavam lesões cutâneas e, ou mucosas, compatíveis com LTA e que foram confirmadas laboratorialmente empregando os métodos relacionados a seguir: pesquisa direta de amastigotas, cultura para leishmânia, inoculação em hamster, intradermoreação de Montenegro, pesquisa indireta de anticorpos fluorescentes e exame histopatológico. Foi realizada também, identificação do parasito pela técnica de anticorpos monoclonais.

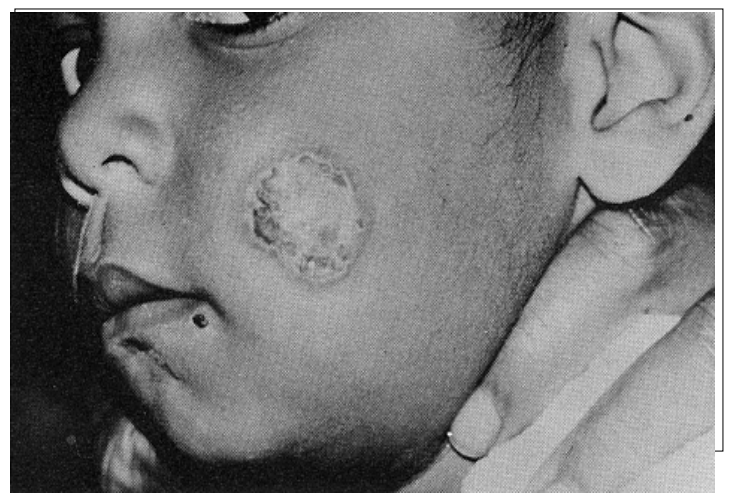

Figura 2 - Lesão na face da mesma criança da Figura 1, em regressão durante o tratamento com antimonial pentavalente.

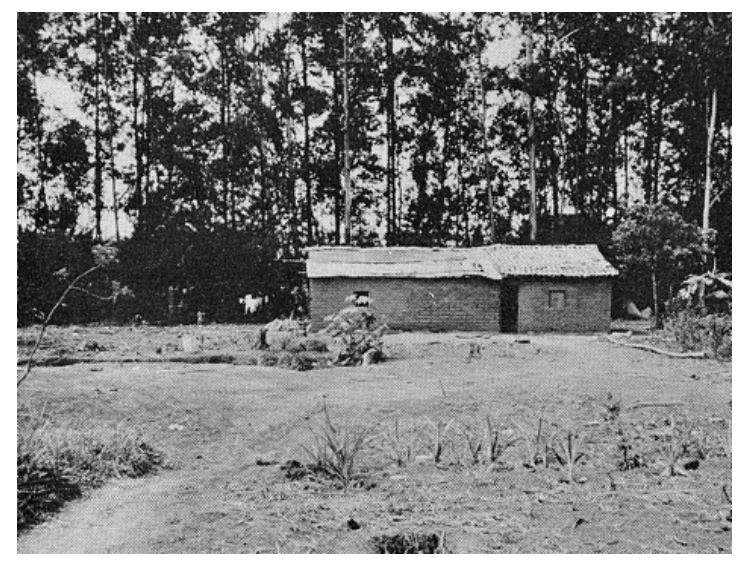

Figura 3 - Residência da criança da Figura 1, com pequena mata de eucalipto e cerrado nos seus arredores. 


\section{RESULTADOS}

A Tabela 1 mostra os aspectos clínicos dos 11 pacientes. Trata-se de quatro mulheres e sete homens, com idades que variam de um a 57 anos. Seis dos 11 , residem na cidade satélite de Planaltina. Todos apresentavam manifestação de úlceras cuja evolução variou de dois a seis meses. Um deles apresentava lesão mucosa no momento do diagnóstico. Os membros inferiores foram acometidos em sete casos, superiores em dois e face em um (Tabela 1). Todos tiveram IRM positiva. Em dez deles pelo menos um dos três exames parasitológicos foi positivo (esfregaço, cultura, inoculação em hamster). Dois deles, tiveram a cepa identificada como Leishmania (Viannia) braziliensis. Nove foram tratados com antimonial pentavalente (20mg SbV/Kg/dia, 20 a 30 dias) e dois com pentamidina $(4 \mathrm{mg} / \mathrm{kg} / \mathrm{dia}$, três doses em dias alternados), sendo acompanhados por um período que variou de um a dez anos. Houve a ocorrência de duas recidivas (Tabela 2).

Tabela 1 - Aspectos clínicos dos doentes com LTA procedentes do DF.

\begin{tabular}{|c|c|c|c|c|}
\hline No/Sexo & Idade (anos) & Residência & Tempo (meses) & Localização \\
\hline $1 / \mathrm{F}$ & 34 & Planaltina & $2 / 4$ & perna D \\
\hline $2 / \mathrm{M}$ & 57 & Planaltina & 3 & perna E \\
\hline $3 / \mathrm{M}$ & 48 & Sobradinho & 2 & braço D \\
\hline $4 / F$ & 11 & Gama & 2 & perna D \\
\hline $5 / F$ & 32 & Paranoá & 4 & coxa D \\
\hline $6 / M$ & 41 & Planaltina & 5 & perna $\mathrm{E}$ \\
\hline $7 / \mathrm{M}$ & 14 & Lago Sul & 2 & perna D \\
\hline $8 / \mathrm{M}$ & 1 & Planaltina & 6 & face \\
\hline $9 / \mathrm{M}$ & 31 & R. das Emas & 5 & braço D/nariz \\
\hline $10 / \mathrm{M}$ & 22 & Planaltina & 6 & antebraço E/face \\
\hline $11 / \mathrm{M}$ & 53 & Planaltina & 4 & perna $\mathrm{E}$ \\
\hline
\end{tabular}

Tabela 2 - Exames laboratoriais, resultado do tratamento e período de acompanhamento dos doentes com LTA procedentes do DF.

\begin{tabular}{lcccc}
\hline$N^{\circ}$ & Amastigota (esf., cult.ou inoc.) & IFA & Tratamento & Resultado/anos \\
\hline 1 & - & $1: 320$ & $\mathrm{SbV}$ & $\mathrm{R} / 3$ \\
2 & + & $1: 320$ & $\mathrm{SbV}$ & $\mathrm{C} / 3$ \\
3 & + & $1: 20$ & $\mathrm{SbV}$ & $\mathrm{C} / 3$ \\
4 & + & $1: 320$ & $\mathrm{SbV}$ & $\mathrm{C} / 1.5$ \\
5 & + & $1: 20$ & $\mathrm{C} / 3$ \\
6 & + & $1: 80$ & $\mathrm{SbV}$ & $\mathrm{C} / 10$ \\
7 & + & $\mathrm{SbV}$ & $\mathrm{C} / 1$ \\
8 & + & peg. & $\mathrm{C} / 1$ \\
9 & + & $1: 40$ & $\mathrm{SbV}$ & $\mathrm{C} / 1$ \\
10 & - & $1: 80$ & $\mathrm{SbV}$ & $\mathrm{C} / 1$ \\
11 & + & $1: 20$ & pentamidina & $\mathrm{R} / 4$ \\
\hline
\end{tabular}

esf. = esfregaço; cult. $=$ cultura; inoc. $=$ inoculação em hamster; $\mathrm{C}=$ cura; $\mathrm{R}=$ recidiva; $\mathrm{SbV}=$ antimônio penta.

\section{DISCUSSÃO}

Oficialmente não há registro pelo MS de casos de leishmaniose tegumentar americana autóctones no Distrito Federal, embora Sampaio et al em 1980 e 1989 tenham relatado dois casos de origem suspeita do Distrito Federal ${ }^{18}{ }^{21}$. Para admitirmos que nossos pacientes possam ter contraído LTA no DF, observamos rigorosamente o pré requisito de incluirmos apenas aqueles que informassem, através de sua história, não haverem saído dos limites do DF por um tempo mínimo de seis meses. O período de incubação da LTA é considerado variável. A observação de casos de leishmaniose tegumentar americana em populações fechadas e com identificação do momento de inoculação, informa período de incubação de dez a 60 dias $^{23}$. A infecção experimental em animais inoculados 
com Leishmania (V) braziliensis demonstrou que o tempo de incubação pode atingir quatro meses em macaco (cinocéfalo) ${ }^{1}$, enquanto macacos Rhesus desenvolveram a doença dentro de 30 a 45 dias $^{6}$. Fonseca, em 1928, descreveu a infecção em cães, tendo período de incubação de 50 a 60 dias $^{7}$, após o qual surgiu a lesão inicial, geralmente uma pápula eritematosa. Então, como pudemos observar, o tempo entre a inoculação da leishmânia e o aparecimento da lesão no hospedeiro tem sido citado na literatura, inferior a 6 meses. Reforçando a suspeita da autoctonia destes casos, temos o paciente de $\mathrm{n}^{\circ} 8$ (Tabela1), com idade de um ano, que nunca saiu da área do DF.

As manifestações clínicas dos pacientes citados são similares às clássicas descrições feitas pelos autores da nossa literatura. A idade variou de um a 57 anos de idade, tendo sido acometidas três crianças (até os 14 anos de idade) em um total de 11 casos. Este número é proporcionalmente maior que o percentual de $14 \%$ obtido em nosso estudo anterior, neste mesmo hospital, considerando a faixa etária de um a 20 anos $^{18}$. Certamente o acometimento na infância realça a suspeita da presença do transmissor no peridomicílio, forma de transmissão mais comum nas áreas peri-urbanas.

Quanto ao diagnóstico, $9(81,81 \%)$ de 11 pacientes tiveram pelo menos um dos exames parasitológicos positivo (pesquisa de amastigota no esfregaço, cultura ou inoculação de hamster). Seis de 11 (54,54\%), tiveram a imunofluorescência indireta positiva. Estes resultados comportaramse semelhante aos dados da literatura ${ }^{5}$.

Dois dos 11 pacientes referidos, tiveram os parasitos identificados por anticorpos monoclonais como Leishmania ( $V$ ) braziliensis. O paciente de no 9 era portador da forma mucosa da doença e o paciente de $\mathrm{n}^{0} 11$ (Tabela 1) acompanhado por 10 anos, teve recidiva da doença com lesões na mucosa nasal. Logo, achamos que a espécie predominante possa ser a Leishmania (V) braziliensis, tendo em vista o seu tropismo pelas mucosas.

Quanto ao tratamento, usou-se na maioria dos pacientes o esquema clássico com antimoniais pentavalentes e dois foram submetidos a tratamento com pentamidina, droga de eficácia já comprovada na leishmaniose ${ }^{322}$. Houve duas recidivas após o tratamento clássico com os antimoniais, fato que também pode ocorrer com mais frequência na LTA causada por $L(V) b^{19}$.

Em relação à transmissão da LTA, há dois padrões epidemiológicos bem definidos. O primeiro deles está associado à derrubada das matas, onde os reservatórios são animais silvestres, e o segundo ocorre em áreas onde não existe desmatamento e animais peridomiciliares como cães, eqüinos e roedores parecem constituir os reservatórios ${ }^{25}$. A descrição da doença em cães, data do início do século (1913), mas este fato era considerado como tendo o cão sido acometido acidentalmente ao penetrar na floresta. Recentemente, é citada alta prevalência da doença em cães e eqüinos principalmente no Brasil e Venezuela. Com as mudanças das condições ambientais acredita-se que tenha havido uma adaptação de alguns vetores, como Lutzomyia intermedia ${ }^{10}$ que passaram a viver em torno das residências levando o homem a adquirir a infecção peridomiciliar. Nas duas últimas décadas têm sido descritos, em vários estados do Brasil, surtos nas áreas peri-urbanas das grandes cidades ${ }^{161726}$. A principal espécie transmissora da leishmaniose cutânea é o Lutzomyia intermedia, cuja distribuição tem sido pouco estudada ${ }^{12}$. Vexenat, em 1991, estudando a fauna flebotomínica do DF encontrou o vetor Lutzomyia whitmani largamente distribuído na sua área ${ }^{27}$. Apesar de não parecer existir condições bastante satisfatórias para a ocorrência da LTA em Brasília, pois esta é uma cidade de baixo índice pluviométrico e baixa umidade, portanto, parece, sem grandes favorecimentos de criadouros biológicos dos flebotomíneos, entretanto, existe aqui vegetação tipo mata do cerrado entrecortada por pequenas matas ciliares, onde há roedores que poderiam estar envolvidos na transmissão da doença.

A maioria dos pacientes portadores de LTA atendidos aqui no DF, procede de Goiás, MG, Bahia e Estados do Norte e Nordeste ${ }^{20}$. O DF está localizado dentro do estado de Goiás e faz também fronteira com Minas Gerais, dois estados com altos índices de ocorrência de LTA. Houve um crescimento populacional descontrolado e exagerado do DF. Segundo o censo de 1991, a população que era de 1.598415 habitantes, em 1996 totalizava 1.821946 (IBGE). Isto representa um crescimento superior a 220 mil habitantes em 5 anos. Grande parte desta população vinda de outros estados, aqui se assentaram e se instalaram em condições inadequadas de saúde e saneamento gerando grandes alterações sociais. Fazendo parte desta população, podem ter migrado pacientes com leishmaniose que aqui se instalaram, inclusive com seus animais de estimação, numa convivência com potenciais 
transmissores da doença, já encontrados no $\mathrm{DF}^{27}$, facilitando desta forma a infeção peridomiciliar.

Por todos estes fatores, acreditamos que os pacientes relacionados aqui, possam ter adquirido
LTA no DF, mas, para confirmarmos a autoctonia destes casos é necessário estudar a possível infecção nos reservatórios, flebotomíneos e na população e isto já é objeto de um projeto para futuro estudo.

\section{AGRADECIMENTOS}

Ao Sr. Tércio Rodrigues, Técnico do Laboratório de Dermatomicologia da UnB, pelo seu empenho e ajuda na realização do trabalho. Ao Prof. J.J. Shaw do Instituto Evandro Chagas, FNS-Belém,
Pará, pela identificação das espécies de leishmânia. Ao Dr. Cyro Coimbra de Resende e Sr. João Afonso S. Sobrinho da Fundação Nacional da Saúde - DF, MS, pela disponibilidade na ajuda.

\section{REFERÊNCIAS BIBLIOGRÁFICAS}

1. Amaral ADF. Contribuição para o estudo da inoculação experimental da Leishmania brasiliensis. Anais da Faculdade de Medicina da Universidade de São Paulo 17:303-355, 1941.

2. Azulay RD, Salgado U. Surto epidêmico de leishmaniose tegumentar americana observado em paraquedistas do exército na Amazônia. Medicina Cutânea 4:347-352, 1966.

3. Correia D, Macêdo VO, Carvalho EM, Barral A, Magalhães AV, Abreu MVA, Orge MGO, Marsden PD. Estudo comparativo entre antimoniato de meglumina, isotianato de pentamidina e sulfato de aminosidine, no tratamento de lesões cutâneas primárias causadas por Leishmania (Viannia) braziliensis. Revista da Sociedade Brasileira de Medicina Tropical 29:447-453, 1996

4. Costa JML. Leishmaniose tegumentar americana:origens e histórico no Brasil. Acta Amazônica 22:71-77, 1992.

5. Cuba CAC, Marsden PD, Barreto AC, Rocha R, Sampaio RNR, Patzlaff L. Parasitologic and immunologic diagnosis of American (Mucocutaneous) leishmaniasis. Bulletin Panamerican Health Organization 15:249-259, 1981.

6. Cunha AM. Infecções experimentais na leishmaniose tegumentar americana. Memórias do Instituto Oswaldo Cruz 41:263-282, 1944.

7. Fonseca F. Infecção experimental do cão por cultura de Leishmania brasiliensis. Anais da Faculdade de Medicina da Universidade de São Paulo 3:53-57, 1928.

8. Fundação Nacional de Saúde, Centro Nacional de Epidemiologia. Informe Epidemiológico do SUS, Ministério da Saúde, Ano VI:59-60, 1997

9. Furtado TA, Aleixo J, Lopes CF. Surto de leishmaniose tegumentar americana em Minas Gerais. O Hospital 70:259-266, 1966.

10. Gomes AC, Rabello EX, Santos JLF, Galati EAB. Aspectos ecológicos da leishmaniose tegumentar americana. 2 Ecótopo artificial como abrigo de Psychodopygus intermedius e observações sobre alimentação e reprodução sob influência de fatores físicos naturais. Revista de Saúde Pública 16:149-159, 1982.

11. Jones TC, Jonhson WD, Barreto AC, Lago E, Badaró R, Cerf B, Reed G, Netto EM, Tada MS, Franca F, Wiese K,
Golightly L, Fikrig E, Costa JM, Cuba CC, Marsden PD. Epidemiology of American Cutaneous Leishmaniasis due to Leishmania braziliensis braziliensis. The Journal of Infectious Diseases 156:73-83, 1987.

12. Lainson R. The American Leishmaniasis:Some observations on their ecology and epidemiology. Transactions Royal Society of Tropical Medicine and Hygiene 77:569-596, 1983.

13. Marsden PD. Mucosal leishmaniasis (Espúndia/Escomel, 1911). Transaction Royal Society of Tropical Medicine and Hygiene 80:859-76, 1986.

14. Mayrink W, Williams P, Coelho MN, Dias M, Martins NV, Magalhães PA, Costa CA, Falcão AR, Melo MN, Falcão AE. Epidemiology of dermal leishmaniasis in the Rio Doce Valley, State of Minas Gerais, Brazil. Annals of Tropical Medicine and Parasitology 73:123 -127, 1979.

15. Miranda C, Massa L, Marques CCA. Análise da ocorrência de leishmaniose tegumentar americana através de imagem obtida por sensoriamento remoto orbital em localidade urbana da região Sudeste do Brasil. Revista de Saúde Pública 30:433-437, 1996.

16. Oliveira Neto MP, Pirmez C, Rangel E, Schubach A, Grimaldi JR G. An outbreak of american cutaneous leishmaniasis (Leishmania braziliensis braziliensis) in a periurban area of Rio de Janeiro city, Brazil: clinical and epidemiological studies. Memórias do Instituto Oswaldo Cruz 83:427-435, 1988.

17. Sabroza PC. O domicílio como fator de risco na leishmaniose tegumentar americana. Estudo epidemiológico em Jacarepaguá, municipio do Rio de Janeiro. Dissertação de Mestrado da Escola Nacional de Saude Pública-FIOCRUZ. Rio de Janeiro, 1981.

18. Sampaio RNR. Tratamento hospitalar da leishmaniose cutâneo mucosa. Tese de Mestrado, UFMG. Belo Horizonte, 1984.

19. Sampaio RNR, Marsden PD. Mucosal leishmaniasis unresponsive to pentavalent antimonial therapy (Glucantime) sucessfully treated with Ambisome. Transaction Royal Society Tropical Medicine and Hygiene 84:362, 1997.

20. Sampaio RNR, Marsden P D, Furtado T, Barreto AC, Cuba CC, Campbell MG. Avaliação clínica e laboratorial de 114 
casos hospitalares de leishmaniose cutâneo mucosa. Anais Brasileiros de Dermatologia 64:201-206, 1989.

21. Sampaio RNR, Rocha RAA, Marsden PD, Cuba CC, Barreto AC. Leishmaniose tegumentar americana. Casuística do Hospital Escola da UnB. Anais Brasileiros de Dermatologia 55:69-76, 1980.

22. Sampaio RNR, Sampaio JHD, Marsden PD. Pentavalent antimonial treatment in mucosal leishmaniasis. The Lancet 1:1097, 1985.

23. Santos I, Santos IB, Montenegro D, Lemos ER, Pereira T. Uso do itraconazol em 26 pacientes portadores de leishmaniose tegumentar americana. Anais Brasileiros de Dermatologia 70:103-107, 1995.

24. Stolf, HO, Marques AS, Marques MEA, Yoshida ELA, Dillon NL. Surto de leishmaniose tegumentar americana em Itaporanga, São Paulo (Brasil). Revista do Instituto de Medicina Tropical 35:437-442, 1993.

25. Talhari S, Talhari AC, Ferreira LCL, Naiff R. Leishmaniose Cutâneo-mucosa. In:Talhari S, Neves RG (eds) Dermatologia Tropical, $1^{\text {a }}$ edição, Rio de Janeiro, Medsi, p.23-45, 1995.

26. Vasconcelos $A W$, Sousa $A Q$, Vasconconcelos IAB, Lima JEO, Alencar JE. A new and peculiar outbreak of cutaneous leishmaniasis on the metropolitan region of Fortaleza, capital of Ceará State, Brazil. In: Meeting on the epidemiology of cutaneous and mucosal american leishmaniasis, Guiana Francesa, dec. 10-11, 1987.

27. Vexenat JA. Temperatura, um fator determinante na atividade de Lutzomia whitmani (Diptera, Psichodidae), Antunes e Coutinho (1939). Dissertação de Mestrado. Instituto de Ciências Biológicas, Universidade de Brasília. Brasília, 1991. 